\title{
Prognostic significance of AMPK activation in advanced stage colorectal cancer treated with chemotherapy plus bevacizumab
}

\author{
E Zulato ${ }^{1,6}$, F Bergamo ${ }^{2,6}$, A De Paoli ${ }^{3}, \mathrm{G}$ Griguolo ${ }^{2}, \mathrm{G}$ Esposito ${ }^{1}$, G L De Salvo ${ }^{3}$, C Mescoli ${ }^{4}$, M Rugge ${ }^{4}$, \\ M Nardin ${ }^{5}$, L Di Grazia ${ }^{5}$, S Lonardi ${ }^{2}$, S Indraccolo*,1,6 and V Zagonel ${ }^{2,6}$ \\ ${ }^{1}$ Immunology and Molecular Oncology Unit, Istituto Oncologico Veneto-IRCCS, Via Gattamelata 64, 35138 Padova, Italy; ${ }^{2}$ Medical \\ Oncology 1 Unit, Istituto Oncologico Veneto-IRCCS, Via Gattamelata 64, 35138 Padova, Italy; ${ }^{3}$ Clinical Trials and Biostatistics Unit, \\ Istituto Oncologico Veneto-IRCCS, Via Gattamelata 64, 35138 Padova, Italy; ${ }^{4}$ Pathology and Cytopathology Unit, Department of \\ Medicine, University of Padova, Via Gabelli 61, 35121 Padova, Italy and ${ }^{5}$ Radiology Unit, Istituto Oncologico Veneto-IRCCS, \\ Via Gattamelata 64, 35138 Padova, Italy
}

Background: AMP-activated protein kinase (AMPK) has a central role in cellular energy sensing and is activated in preclinical tumour models following anti-vascular endothelial growth factor (VEGF) therapy. The possible predictive or prognostic role of AMPK status in cancer patients treated with anti-VEGF drugs has not been investigated so far.

Methods: Expression of components of the AMPK pathway including phosphorylated AMPK (PAMPK), phosphorylated acetyl-Coa carboxylase (pACC) and liver kinase B1 (LKB1) was investigated by immunohistochemistry in 48 colorectal cancers treated with FOLFIRI plus bevacizumab. Correlation between PAMPK and PACC and associations between the AMPK pathway scores and clinico-pathological characteristics were assessed. Overall survival (OS) was estimated through Kaplan-Meier method, whereas hazard ratios were computed to identify prognostic factors.

Results: Fourteen patients (29.2\%) were included in the pAMPK-negative group (score $\leqslant 5$ ), whereas 34 patients $(70.8 \%$ ) were included in the PAMPK-positive group (score $>5$ ). The Spearman's coefficient for the correlation between pAMPK and pACC scores in primary tumour samples was $0.514(P=0.0002)$. Low pAMPK levels were associated with worse OS ( $P$-value 0.0002$)$ but not with PFS, whereas low pACC levels were associated both with worse OS and PFS ( $P$-value 0.0007 and 0.01 , respectively).

Conclusions: Our findings suggest that high tissue AMPK activation is a prognostic biomarker in this cohort of metastatic colorectal cancer patients.

Colorectal cancer (CRC) is the third most common tumour worldwide and represents the second leading cause of cancer death in Europe and the USA. Approximately $20 \%$ of CRC patients will present distant metastasis (stage IV) at the time of diagnosis with 5 -year survival $<10 \%$ (Jessup et al, 1996). Management of metastatic CRC (mCRC) has substantially evolved over the past decade with the introduction of new chemotherapy combinations and biological agents. The US Food and Drug Administration approved the anti-vascular endothelial growth factor (VEGF) antibody bevacizumab for first-line treatment of patients with mCRC in 2004 (Hurwitz et al, 2004). Since its approval, bevacizumab in combination with chemotherapy has become the standard first-line treatment option in mCRC, extending both progression-free survival (PFS) and overall survival (OS) (Hurwitz et al, 2004; Kabbinavar et al, 2005; Saltz et al, 2008). Despite clinical use of bevacizumab for nearly a decade, however,

*Correspondence: Dr S Indraccolo; E-mail: stefano.indraccolo@unipd.it
${ }^{6}$ These authors equally contributed to this work.

Received 17 January 2014; revised 2 April 2014; accepted 28 April 2014; published online 3 June 2014

(c) 2014 Cancer Research UK. All rights reserved 0007-0920/14 
important questions still remain, including the prediction and management of potential adverse effects and selection of the ideal patients' population. In this regard, predictive biomarkers of response are still not available, although a number of potential systemic, circulating, tissue and imaging biomarkers have emerged from recently completed clinical trials (Jubb and Harris, 2010).

Observations in patients (Faivre et al, 2007; Mehta et al, 2011) and preclinical findings (Nardo et al, 2011) both suggest that VEGF blockade may perturb the energy balance of cancer cells. Several groups have looked at metabolic perturbations associated with anti-angiogenic therapy by using either magnetic resonance spectroscopy (Keunen et al, 2011) or hyperpolarised 13C magnetic resonance spectroscopy (Bohndiek et al, 2012), reporting increased lactate levels and production of labelled malate from fumarate, an early indicator of cell necrosis. In line with these findings, we showed for the first time that anti-VEGF therapy causes metabolic perturbations in experimental tumours, including marked reduction in glucose and ATP levels (Nardo et al, 2011). Moreover, probably as consequence of marked glucose depletion and exhaustion of ATP levels, we also observed that anti-angiogenic therapy increased AMP-activated protein kinase (AMPK) activation in tumour xenografts and that tumour cells lacking AMPK activity undergo marked tumour necrosis following a short-term course of anti-VEGF therapy (Nardo et al, 2011).

AMPK is a heterotrimeric serine/threonine protein kinase, and is activated following its phosphorylation by its upstream kinase liver kinase B1 (LKB1) (also known as STK11) in response to an increase in cellular AMP/ATP ratio (Shackelford and Shaw, 2009). AMPK has a central role in the regulation of energy metabolism in all eukaryotes, and governs glucose and lipid metabolism in response to alterations in nutrients and intracellular energy levels, contributing to maintain the steady-state levels of intracellular ATP (Hardie et al, 2012). Phosphorylation of acetyl-CoA carboxylases by AMPK contributes to regulation of fatty acid metabolism (Hardie et al, 2012) and is considered an indirect readout of AMPK activation. We hypothesise that the integrity of signalling pathways involved in the control of cell metabolism, growth and quiescence, such as AMPK, could be important to sense changes in the tumour microenvironment caused by angiogenesis inhibition and to enable tumour cells adaptation. Accordingly, tumour cells lacking AMPK activity are expected to undergo necrosis following anti-angiogenic therapy, due to their incapacity to maintain ATP levels. Although in patients the association between tumour necrosis and outcome of antiangiogenic therapy is not firmly established, in preclinical models necrosis often leads to increased recruitment into tumours of macrophages and other specialised subsets of myeloid cells which can promote tumour angiogenesis and leads to escape from VEGF blockade (Ferrara, 2010). Therefore, defective AMPK activity might be predicted to associate initially with improved tumour response, followed by increased resistance to VEGF neutralisation.

Several studies investigated the prognostic role of AMPK in human malignancies, including colorectal, lung, ovarian, gastric cancer, renal cell carcinoma and hepatocarcinoma (Baba et al, 2010; Tsavachidou-Fenner et al, 2010; Buckendahl et al, 2011; Kang et al, 2012; William et al, 2012; Zheng et al, 2013). In most of these studies-except gastric cancer (Kang et al, 2012)-low levels of AMPK activation correlated with poor prognosis. With regard to targeted therapy, one study reported that low phosphorylated AMPK (pAMPK) levels are associated with poor response to bevacizumab and interferon- $\alpha$ therapy in renal cell carcinoma (TsavachidouFenner et al, 2010). Based on this rationale and the paucity of studies investigating the predictive value of AMPK activation in patients treated with anti-angiogenic drugs, we designed a pilot retrospective study to analyse activation of the AMPK pathway in CRC patients treated with bevacizumab plus chemotherapy and to correlate it with patient clinical data and survival.

\section{MATERIALS AND METHODS}

Patients and treatments. Study design and tissue analysis were approved by the institutional review board of Istituto Oncologico Veneto. Our study cohort was generated through a query in our patients' files for all patients with mCRC who received between January 2007 to May 2011 FOLFIRI-bevacizumab as a first-line treatment. This regimen consists of bi-weekly cycles of irinotecan $\left(180 \mathrm{mg} \mathrm{m}^{-2}\right)$, bevacizumab $\left(5 \mathrm{mg} \mathrm{kg}^{-1}\right)$ and a bolus of 5-FU $\left(400 \mathrm{mg} \mathrm{m}^{-2}\right)$ followed by a $46 \mathrm{~h}$ continuous infusion of the same drug $\left(2400 \mathrm{mg} \mathrm{m}^{-2}\right)$.

Clinical information was extracted from patients' medical records. Inclusion criteria comprised pathology-confirmed adenocarcinoma of the colon or rectum in a TNM stage IV with availability of samples of the primary carcinoma retrieved before beginning of the anti-angiogenic therapy. A total of 48 patients met all inclusion criteria and comprised the patient study cohort. Collected variables were related to demography, tumour location, number and location of metastasis, their synchronous or metachronous nature, surgery of the primary tumour and of metastasis, adjuvant chemotherapy, KRAS status, Eastern Cooperative Oncology Group (ECOG) performance status (PS) and carcino-embrionic antigen (CEA) levels in the blood at the beginning of first-line therapy.

Radiological response during treatment was evaluated by computerised tomography scan of the chest and abdomen conducted every 2-3 months and was classified using Response Evaluation Criteria In Solid Tumours (RECIST) 1.1 criteria (Eisenhauer et al, 2009). Survival data were obtained from patient medical records. Information on subsequent lines of chemotherapy was also retrieved.

Immunohistochemical staining. Immunohistochemistry (IHC) analysis was conducted in 5 - $\mu$ m-thick paraffin-embedded tumour sections, dewaxed in xylene and re-hydrated in progressively less concentrated alcohol solutions.

To evaluate the status of the AMPK pathway, slides were incubated with rabbit anti-pAMPK monoclonal antibody (Thr172, Cell Signaling Technology, Danvers, MA, USA), anti-phosphorylated acetyl-Coa carboxylase (pACC) polyclonal antibody (Ser79, Cell Signaling Technology) and anti-LKB1 policlonal antibody (Ley 37D/G6, Santa Cruz Biotechnology, Dallas, TX, USA), according to the manufacturer's instructions. Ethylene diaminetetraacetic acid disodium salt dihydrate, $\mathrm{pH} 8$ (1 mM) for $45 \mathrm{~min}$ was used as a standard microwave-based antigen retrieval method for all antigens, except for LKB1. Citric acid buffer, pH 6.0 (10 mM) for $20 \mathrm{~min}$ at $94{ }^{\circ} \mathrm{C}$ was used for LKB1 antigen retrieval. Staining conditions for pAMPK (1:150 dilution) and pACC $(1: 200$ dilution) were previously reported (Nardo et al, 2011). IHC was performed using a DAKO autostainer (Glostrup, Denmark), according to the manufacturer's protocol. For LKB1, after blocking the endogenous peroxidase, tissue sections were incubated with $5 \%$ normal goat serum in $0.1 \%$ Tween-20 tris-buffered saline buffer, pH 7.6 (60 min). Primary anti-LKB1 (1:100) antibody was incubated overnight at $4{ }^{\circ} \mathrm{C}$. Sections were subsequently incubated with biotinylated anti-rabbit or anti-mouse antibody for $30 \mathrm{~min}$, followed by Vectastain ABC reagent (Vector Labs, Peterborough, $\mathrm{UK}$ ) for additional $30 \mathrm{~min}$. Liquid diaminobenzidine (DAB; DAKO) was used as a chromogenic agent for $10 \mathrm{~min}$ and sections were counter-stained with Mayer's haematoxylin. Between each immunostaining step, slides were briefly washed in Tween-20 tris-buffered saline. Specificity of each staining procedure was confirmed by replacing the primary antibody with PBS. Based on previous work (Nardo et al, 2011), sections of IGROV-1 and OC316 tumour xenografts treated with anti-VEGF monoclonal antibody $(\mathrm{mAb})$ were included as positive and negative controls for pAMPK and pACC, respectively (Supplementary Figure 1). 
To setup IHC for LKB1, tumour xenografts formed by A549 cellswhich lack LKB1 expression (Sanchez-Cespedes et al, 2002)-were analysed as a negative control. As a positive control, xenografts formed by A549 cells reconstituted with LKB1 cDNA were analysed (Supplementary Figure 1).

IHC staining was evaluated by two experienced pathologists (GE and $\mathrm{CM}$ ) with no prior knowledge of clinical data. In case of disagreement, results were discussed in a multi-headed microscope, and a consensus was reached. Assessment was conducted at a magnification of $\times 100$, on the basis of the Quick Score Method (Hadad et al, 2009). Immunoreactivity was scored semi-quantitatively for both the intensity and the proportion of cells staining: intensity was given scores $0-3$ (no staining 0 , light staining 1 , moderate staining 2 and strong staining 3 ) and proportion was given scores 1-6 (0-4\% 1, 5-20\% 2, 21-40\% 3, 41-60\% 4, 61-80\% 5 and $81-100 \% 6$ ). The two scores were multiplied to obtain the final score (range 0-18). Concordance between the two observers was evaluated by the weighted kappa coefficient. The weighted kappa coefficient was 0.660 for pAMPK, 0.719 for pACC and 0.9114 for LKB1 score, indicating good agreement between the pathologists. For statistical analysis, samples with pAMPK, pACC or LKB1 score $\leqslant 5$ were grouped in one group and were compared with those with score $>5$. This cutoff was chosen as it was found to best stratify samples with strong/moderate $v s$ light/negative staining.

Clinical endpoints and statistical analysis. Endpoint of the study was to determine the association between pAMPK protein expression and OS or PFS. The association between pACC protein expression and OS or PFS was also assessed. OS was defined as the time from date of first-line treatment to date of death or to last follow-up for censored data. PFS was calculated from the beginning of therapy with FOLFIRI-bevacizumab to the date of first disease progression, or death from all causes or censored at the last documented follow-up date.

To verify the reliability of IHC in assessing the activation of the AMPK pathway, we tested the correlation between $\mathrm{pAMPK}$ and

Table 1. Association between clinico-pathological characteristics of metastatic colorectal cancer patients and immunohistochemical data

\begin{tabular}{|c|c|c|c|c|c|c|c|}
\hline \multirow[b]{2}{*}{ Characteristics } & \multirow[b]{2}{*}{$N(\%)$} & \multicolumn{3}{|c|}{ pACC score } & \multicolumn{3}{|c|}{ pAMPK score } \\
\hline & & $\leqslant 5(N=15)$ & $>5(N=33)$ & $P$-value & $\leqslant 5(N=14)$ & $>5(N=34)$ & $P$-value \\
\hline Gender & & & & 0.751 & & & 1.00 \\
\hline Male & $29(60.4)$ & 10 & 19 & & 8 & 21 & \\
\hline Female & $19(39.6)$ & 5 & 14 & & 6 & 13 & \\
\hline Age at diagnosis (years) & & & & 0.757 & & & 0.756 \\
\hline$\leqslant 65$ & $25(52.1)$ & 7 & 18 & & 8 & 17 & \\
\hline$>65$ & $23(47.9)$ & 8 & 15 & & 6 & 17 & \\
\hline Primary site & & & & 0.724 & & & 0.729 \\
\hline Colon & $32(66.7)$ & 10 & 22 & & 9 & 23 & \\
\hline Rectum & $14(29.2)$ & 3 & 11 & & 3 & 11 & \\
\hline Colon and rectum ${ }^{a}$ & $2(4.2)$ & - & - & & - & - & \\
\hline Site of metastasis & & & & 0.742 & & & 0.328 \\
\hline Liver & $16(33.3)$ & 4 & 12 & & 3 & 13 & \\
\hline Other & $32(66.7)$ & 11 & 21 & & 11 & 21 & \\
\hline Synchronous metastasis & & & & 0.239 & & & 1.00 \\
\hline Yes & $39(81.2)$ & 14 & 25 & & 12 & 27 & \\
\hline No & 9 (18.7) & 1 & 8 & & 2 & 7 & \\
\hline KRAS wildtype & & & & 0.755 & & & 1.00 \\
\hline Yes & $22(45.8)$ & 8 & 14 & & 6 & 16 & \\
\hline No & $24(50.0)$ & 7 & 17 & & 7 & 17 & \\
\hline Unknown $^{\mathrm{a}}$ & $2(4.2)$ & - & - & & - & - & \\
\hline ECOG-PS & & & & 0.031 & & & 0.294 \\
\hline 0 & $36(75.0)$ & 8 & 28 & & 9 & 27 & \\
\hline 1 & $12(25.0)$ & 7 & 5 & & 5 & 7 & \\
\hline Ablation/resection of metastasis & & & & 0.004 & & & 0.206 \\
\hline Yes & $28(58.3)$ & 4 & 24 & & 6 & 22 & \\
\hline No & $20(41.7)$ & 11 & 9 & & 8 & 12 & \\
\hline Lines of treatment & & & & 1.00 & & & 1.00 \\
\hline 1 & $9(18.7)$ & 3 & 6 & & 3 & 6 & \\
\hline$\geqslant 2$ & 39 (81.2) & 12 & 27 & & 11 & 28 & \\
\hline Carcino-embrionic antigen $>30$ & & & & 1.00 & & & 0.321 \\
\hline Yes & $16(33.3)$ & 5 & 11 & & 3 & 13 & \\
\hline No & $31(64.6)$ & 10 & 21 & & 11 & 20 & \\
\hline Unknown $^{\mathrm{a}}$ & $1(2.1)$ & - & - & & - & - & \\
\hline
\end{tabular}


pACC scores on the same tumour sample, using the Spearman's coefficient. The statistical significance of association between pACC/pAMPK score $(\leqslant 5$ vs $>5)$ and clinical-pathological data was assessed by Fisher's exact test.

The survival probability was estimated by means of the KaplanMeier method, and heterogeneity in survival among strata of selected variables was assessed through the log-rank test. A multivariate Cox proportional hazards model was applied to identify factors that were associated with the risk of death. A Collett's Model Selection approach (Collett, 1994) was used with a level of significance of 0.2 at univariate analysis and stay and entry criterions of 0.1 to build up multivariate models. To check the proportional hazards assumption, a score process (which is a transformed partial sum process of the martingale residuals) was compared with the simulated processes under the null hypothesis that the proportional hazards assumption holds (Lin et al, 1993). Multivariate hazard ratios (HRs), with their 95\% confidence intervals (CIs), were computed. Two separate models were performed as the correlation between pACC and pAMPK scores did not allow to include them in a single model simultaneously. The Kaplan-Meier method was computed to estimate the survival probability from progression to death or last follow-up. Data analyses were performed using the SAS statistical package (SAS, release 9.2; SAS Institute, Cary, NC, USA).

\section{RESULTS}

Patient characteristics. Clinical data of the 48 patients enrolled in this study are listed in Table 1 . The mean (median) age at diagnosis was $63.5(64.9)$ years with a range from 28 to 74 years, and 29 patients $(60.4 \%)$ were male. The mean (median) age at first-line treatment of patients was $64.2(66.0)$ years with a range from 29 to 76 years. Fourteen patients $(29.2 \%)$ had rectal cancer, whereas thirty-two patients $(66.7 \%)$ had colon cancer. Two patients presented a concomitant double primary tumour of the colon and rectum. Thirty-nine patients $(81.2 \%)$ had metastatic disease at diagnosis, whereas nine patients (18.7\%) developed metachronous metastases. Sixteen patients $(33.3 \%)$ had exclusively liver metastases. Thirty-six patients $(75.0 \%)$ had a performance status of 0 when first-line chemotherapy began. Blood levels of CEA were evaluated before starting chemotherapy, showing an average value of $291(0.5-6762) \mu \mathrm{gl}^{-1}$. KRAS status was assessed on tumour samples from 46 patients and 24 of them (52.2\%) presented mutations in exons 12 or 13 . Twenty-eight patients $(58.3 \%)$ underwent surgery of metastases or loco-regional treatment with radical intent (such as microwaves or radiofrequencies of hepatic lesions). Thirty-nine patients underwent two or more lines of chemotherapy (including re-challenge with the same drugs) after progression under FOLFIRI-bevacizumab treatment.

pAMPK and pACC expression in CRC. We performed IHC of pAMPK to investigate the LKB1/AMPK pathway activation in tumour sections. Phosphorylation of acetyl-CoA carboxylases, a direct downstream target of AMPK, was also analysed. In general, pACC and pAMPK expression was detected in the cytoplasm of tumour cells (Figure 1). The Spearman's coefficient for the correlation between $\mathrm{pAMPK}$ and $\mathrm{pACC}$ scores in primary tumour samples was $0.514(P=0.0002)$.

Fourteen patients $(29.2 \%)$ were included in the pAMPKnegative group (score $\leqslant 5$ ), whereas thirty-four patients $(70.8 \%$ ) were included in the pAMPK-positive group (score $>5$ ). Moreover, there were $15(31.2 \%)$ and $33(68.7 \%)$ patients in the pACCnegative (score $\leqslant 5$ ) and -positive (score $>5$ ) groups, respectively. A very good correlation between pACC and pAMPK scores was also observed in metastatic tumour samples (Spearman's coefficient $=0.848, \quad P<0.0001)$. However, due to the limited number of paired samples available $(n=17)$, we were unable to investigate the predictive/prognostic value of the pAMPK and pACC scores assessed in metastasis.

We found no statistically significant association between age, gender, primary and metastatic tumour site, KRAS status, CEA blood levels, number of lines of chemotherapy and the high- and low-pAMPK or pACC expression groups (Table 1). A significant association was found between pACC score and surgery of metastasis, as a higher number of patients underwent surgery in the pACC-positive compared with the pACC-negative group, and between pACC score and ECOG performance status (Table 1).

LKB1 expression in CRC. We next investigated whether samples lacking pAMPK expression showed alterations in LKB1, the kinase upstream of AMPK in mammalian cells (Shackelford and Shaw, 2009). Due to the limited amount of samples available, analysis was limited to 11 tumour samples disclosing low levels of pAMPK expression and to additional three samples with high levels of pAMPK expression. By using the same scoring system used for

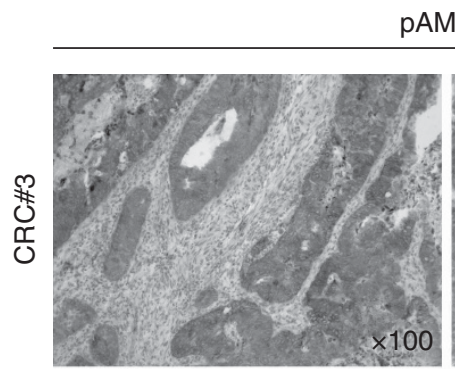

AMPK
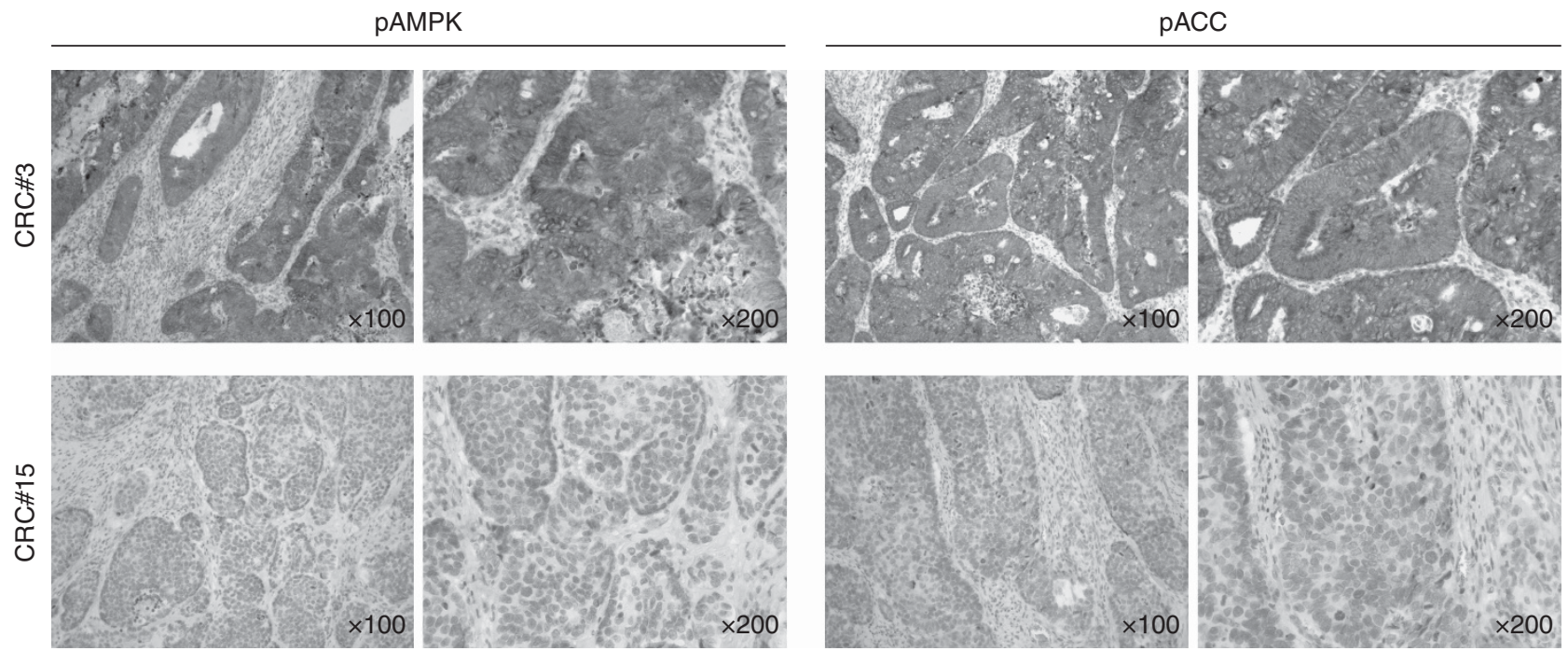

Figure 1. Patterns of phosphorylated AMPK and acetyl-CoA carboxylases expression in primary CRC samples. Representative microphotographs of PAMPK and pACC expression in one strongly positive (CRC\#3) and one negative (CRC\#15) CRC sample; $\times 100$ and $\times 200$ magnifications are shown. 
pAMPK and pACC, we found low LKB1 expression (score $\leqslant 5$ ) in 8 out of 11 samples analysed, whereas the remaining three samples showed moderate LKB1 expression (Figure 2). However, completely absent LKB1 expression was found only in two samples. Conversely, LKB1 was expressed at moderate-high levels in all samples showing high PAMPK that were included in this analysis
(Figure 2). In conclusion, reduced LKB1 expression is found in the majority of CRC samples bearing low pAMPK levels.

Survival according to pAMPK and pACC expression. Among the forty-eight patients, forty-six disease progressions (95.8\%) were documented and twenty-six patients (54.2\%) died during
A
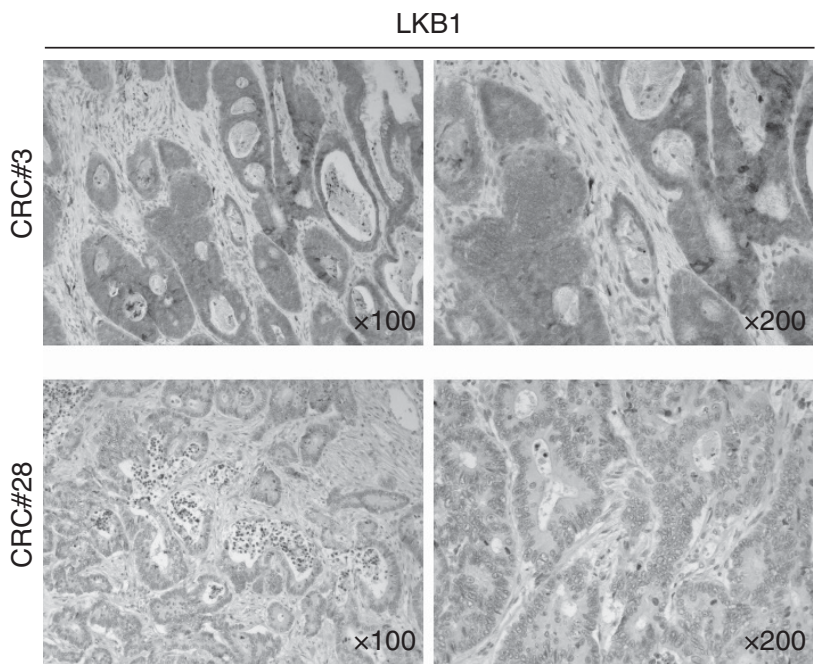

B

\begin{tabular}{|c|c|c|}
\hline & $\begin{array}{l}\text { pAMPK } \\
\text { score }\end{array}$ & $\begin{array}{l}\text { LKB1 } \\
\text { score }\end{array}$ \\
\hline \multicolumn{3}{|l|}{ pAMPK $\leqslant 5$} \\
\hline CRC\#7 & 2 & 5 \\
\hline CRC\#12 & 0 & 4 \\
\hline CRC\#14 & 1 & 3 \\
\hline CRC\#15 & 0 & 6 \\
\hline CRC\#28 & 0 & 0 \\
\hline CRC\#32 & 0 & 5 \\
\hline CRC\#43 & 0 & 3 \\
\hline CRC\#45 & 5 & 6 \\
\hline CRC\#47 & 2 & 0 \\
\hline CRC\#49 & 4 & 6 \\
\hline CRC\#54 & 0 & 2 \\
\hline \multicolumn{3}{|l|}{ pAMPK $>5$} \\
\hline CRC\#3 & 15 & 15 \\
\hline CRC\#29 & 18 & 18 \\
\hline CRC\#51 & 18 & 8 \\
\hline
\end{tabular}

Figure 2. Patterns of LKB1 expression in primary CRC samples. (A) Representative microphotographs of LKB1 expression in samples scoring positive (CRC\#3) or negative (CRC\#28) for PAMPK; $\times 100$ and $\times 200$ magnifications are shown. (B) LKB1 and pAMPK scores in samples disclosing negative (score $\leqslant 5$ ) or positive (score $>5$ ) PAMPK expression.
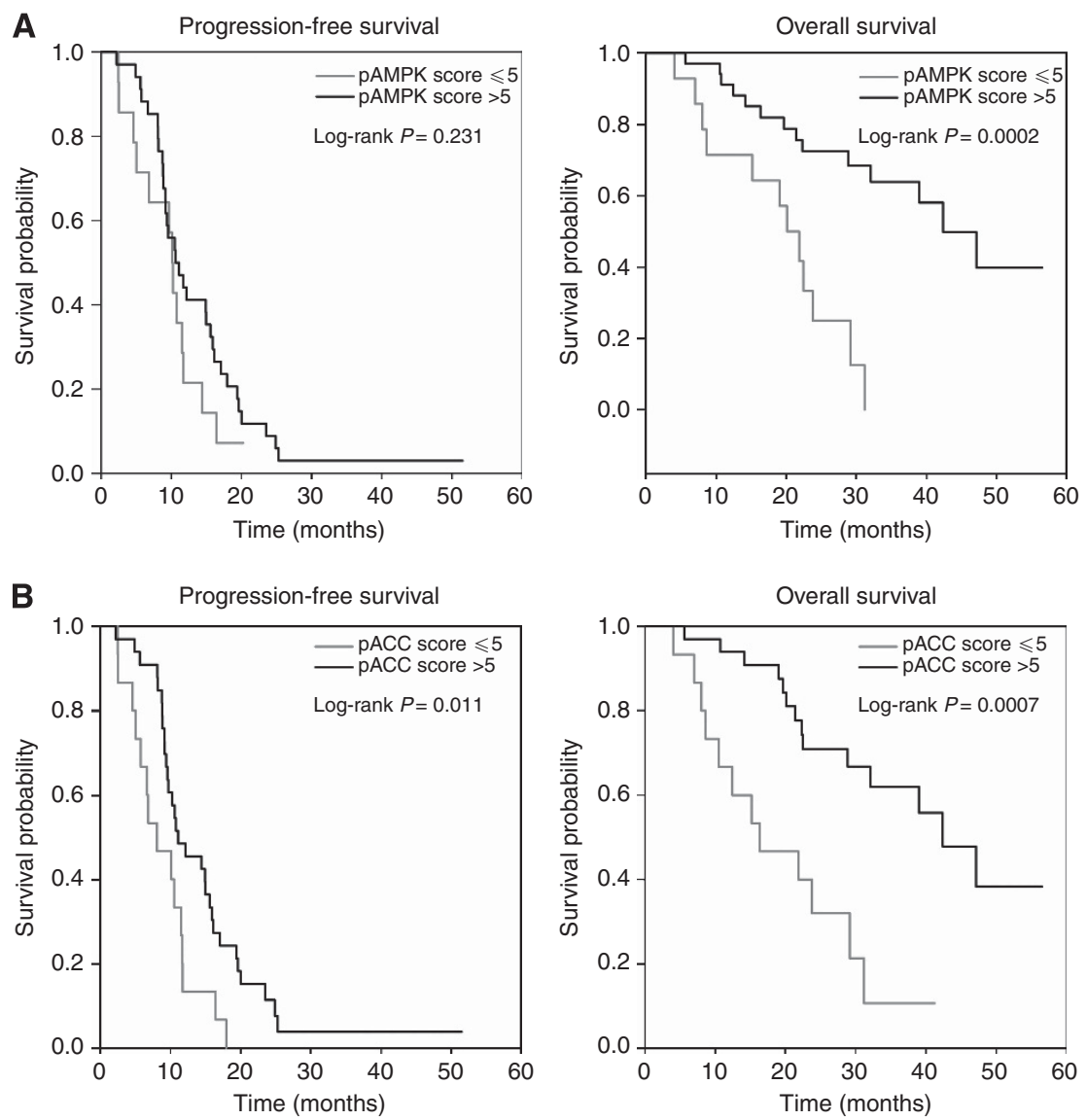

Figure 3. Survival cumulative probabilities by PAMPK or pACC score. Kaplan-Meier PFS (left panel) and OS (right panel) curves for patients who had positive vs negative PAMPK (A) or PACC (B) score. 
follow-up. With a median follow-up of 24.5 months (4.0-56.5), the median PFS was 10.6 months (2.2-51.5), whereas median OS was 32.9 months (12.2-56.5); estimated 1-year OS rate was $85.4 \%$ (95\% CI: 71.8-92.8\%). Low expression of pAMPK was associated with worse OS. Estimated 1-year OS for pAMPK-negative patients was 71.4\% (95\% CI: 40.6-88.2\%), whereas for pAMPK-positive patients was $91.2 \%$ (95\% CI: 75.1-97.1\%). Although patients with pAMPK-positive tumours showed a significantly improved OS compared with patients with pAMPK-negative tumours $(P=0.0002)$, pAMPK status was not significantly associated with PFS $(P=0.23)$ (Figure 3A). Consistently with these results, low pACC expression was associated with worse OS. Estimated 1-year OS for patients with low pACC expression was 66.7\% (95\% CI: 37.5-84.5\%) vs 93.9\% (95\% CI: 77.9-98.4\%) for patients with high pACC expression $(P=0.0007)$. Moreover, pACC status was significantly associated with PFS $(P=0.01)$, as low pACC expression was associated with shorter PFS (Figure 3B).

Among other parameters considered, ECOG performance status $(P=0.001)$, resection of metastases $(P=0.001)$, multiple lines of chemotherapy $(P=0.050)$ and low blood CEA levels $(P=0.004)$ were associated with higher estimated 1-year OS (Table 2).

Multivariate analysis, performed on 47 patients with all data available, disclosed that pAMPK-negative samples were associated with at higher risk of death than pAMPK-positive samples ( $\mathrm{HR}=6.53 ; 95 \% \mathrm{CI}: 2.61-16.37 ; P<0.0001)$. Risk of death was also related with ECOG performance status $(\mathrm{HR}=2.70,95 \% \mathrm{CI}$ : 1.13-6.48; $P=0.026)$, baseline CEA blood values ( $\mathrm{HR}=3.35,95 \%$ CI: $1.38-8.15 ; P=0.008)$ and number of lines of treatment received $(\mathrm{HR}=4.21 ; 95 \% \mathrm{CI}: 1.49-11.94 ; P=0.007)$ (Table 3$)$.

Furthermore, low pACC expression was associated with higher risk of death $(\mathrm{HR}=4.34$; $95 \% \mathrm{CI}$ : $1.86-10.17 ; P=0.0007)$ as were other parameters, including baseline CEA blood value $(\mathrm{HR}=3.56$, 95\% CI: $1.56-8.13 ; P=0.003)$ and lines of treatment received $(\mathrm{HR}=4.15 ; 95 \% \mathrm{CI}: 1.52-11.38 ; P=0.006)$ (Table 3$)$. Resection of metastasis and ECOG performance status were not included in this model, because correlation between variables was detected $(P=0.004$ and $P=0.031$, respectively).

\section{DISCUSSION}

Angiogenesis is required for growth of solid tumours and its inhibition through pharmaceutical blockade of the VEGF pathway is a therapeutic strategy clinically validated in various human malignancies (Heath and Bicknell, 2009). In spite of much efforts, however, the fine mechanisms of tumour regression caused by angiogenesis inhibition remain substantially unknown and validated biomarkers aiding selection of those patients who are most likely to benefit from anti-angiogenic treatment are currently not available (Jubb and Harris, 2010). Previous studies mainly focused on expression of circulating angiogenesis-related biomarkers, such as plasma levels of short VEGF-A isoforms or modified expression of VEGF receptors (VEGFR1 or NRP1) either in plasma or in tumours or genetic markers, including polymorphisms of VEGF-A, VEGFR1, VEGFR2 and IL-8 (reviewed in: (Lambrechts et al, 2013)). Among tumour-related biomarkers, genotyping KRAS and $B R A F$ mutations in CRC has been performed but failed to reveal any differential effects (Price et al, 2011).

Our study was grounded on the assumption that the capacity of tumour cells to adapt to metabolic changes could influence the outcome of anti-angiogenic therapy. Based on preclinical studies by our group, which showed that anti-VEGF therapy causes marked reduction in glucose levels in the tumour microenvironment followed by activation of the energy sensor AMPK (Nardo et al, 2011), we investigated the possible impact of AMPK activation on the response to chemotherapy plus bevacizumab in
Table 2. Estimated 1-year overall survival and $95 \%$ confidence intervals by patient characteristics

\begin{tabular}{|c|c|c|c|c|}
\hline Characteristics & $N(\%)$ & N. failed & $\begin{array}{c}\text { 1-year overall } \\
\text { survival } \\
(95 \% \mathrm{Cl})\end{array}$ & $P$-value \\
\hline Gender & & & & 0.964 \\
\hline Male & 29 & 15 & 82.7 (63.4-92.4) & \\
\hline Female & 19 & 11 & 89.5 (64.1-97.2) & \\
\hline
\end{tabular}

Age at diagnosis (years) $\leqslant 65$ $>65$

Primary site

Colon

Rectum

Colon and rectum ${ }^{\mathrm{a}}$

Site of metastasis

Liver

\section{Synchronous} metastasis

Yes
No

\section{KRAS wildtype}

Yes

No

Unknown ${ }^{a}$

ECOG-PS

0

\begin{tabular}{l|l}
1 & 12
\end{tabular}

Ablation/ resection

metastasis

Yes

No

Lines of treatment

1

\begin{tabular}{|l|r|r}
$\geqslant 2$ & 39 & 20 \\
\hline Score pACC & & \\
\hline$\leqslant 5$ & 15 & \\
$>5$ & 33 & \\
\hline
\end{tabular}

Score pAMPK

$\leqslant 5$

$>5$

Carcino-embrionic

antigen $>30$

Yes

No

Unknown ${ }^{\text {a }}$

\begin{tabular}{|l|l|} 
& \\
\hline 25 & 13 \\
\hline
\end{tabular}

Abbreviations: $\quad \mathrm{Cl}=$ confidence interval; $\mathrm{ECOG}-\mathrm{PS}=\mathrm{ECOG}$ performance status: PAMPK $=$ phosphorylated AMPK; $\mathrm{pACC}=$ phosphorylated acetyl-Coa carboxylase.

${ }^{\mathrm{a}}$ Excluded from the analysis.

cancer patients. We focused on CRC because inhibition of tumour angiogenesis through bevacizumab represents a cornerstone in the treatment of mCRC, extending both PFS and OS when used in association with chemotherapy in first or second line of treatment (Hurwitz et al, 2004; Kabbinavar et al, 2005; Saltz et al, 2008). With regard to AMPK activity, although genetic defects involving the upstream kinase LKB1 are uncommon in this malignancy (Cancer Genome Atlas Network, 2012), a previous retrospective study on population databases reported expression of pAMPK in $57 \%$ of CRC samples (Baba et al, 2010), suggesting that this pathway could be epigenetically silenced in a subset of tumour samples. This 


\begin{tabular}{|c|c|c|c|c|c|}
\hline Characteristics & $\begin{array}{c}\text { Hazard ratio } \\
(95 \% \mathrm{Cl})\end{array}$ & $P$-value & Characteristics & $\begin{array}{l}\text { Hazard ratio } \\
(95 \% \mathrm{Cl})\end{array}$ & $P$-value \\
\hline Score pAMPK & & $<0.0001$ & Score pACC & & 0.0007 \\
\hline $\begin{array}{l}\leqslant 5 \\
>5\end{array}$ & $\begin{array}{c}6.53(2.61-16.37) \\
1\end{array}$ & & $\begin{array}{l}\leqslant 5 \\
>5\end{array}$ & $\begin{array}{c}4.34(1.86-10.17) \\
1\end{array}$ & \\
\hline ECOG-PS & & 0.026 & & & \\
\hline $\begin{array}{l}1 \\
0\end{array}$ & $\begin{array}{c}2.70(1.13-6.48) \\
1\end{array}$ & & & & \\
\hline Carcino-embrionic antigen $>30$ & & 0.008 & Carcino-embrionic antigen $>30$ & & 0.003 \\
\hline $\begin{array}{l}\text { Yes } \\
\text { No }\end{array}$ & $\begin{array}{c}3.35(1.38-8.15) \\
1\end{array}$ & & $\begin{array}{l}\text { Yes } \\
\text { No }\end{array}$ & $\begin{array}{c}3.56(1.56-8.13) \\
1\end{array}$ & \\
\hline Lines of treatment & & 0.007 & Lines of treatment & & 0.006 \\
\hline $\begin{array}{l}1 \\
\geqslant 2\end{array}$ & $\begin{array}{c}4.21(1.49-11.94) \\
1 \\
\end{array}$ & & $\begin{array}{r}1 \\
\geqslant 2 \\
\end{array}$ & $\begin{array}{c}4.15(1.52-11.38) \\
1 \\
\end{array}$ & \\
\hline
\end{tabular}

study, however, did not analyse expression of other components of the pathway, such as pACC or LKB1, nor did it evaluate the possible predictive role of these biomarkers in response to antiVEGF therapy.

Low expression of either pAMPK or pACC in the primary tumour significantly correlated with OS both in univariate and multivariate analyses, suggesting a prognostic role of AMPK pathway activation in advanced stage CRC. This result is in line with the observation that expression of these two markers in primary tumours showed a moderate correlation (Spearman's coefficient $=0.514 ; P=0.0002)$. Moreover, this finding is consistent with preclinical data suggesting a tumour suppressor role for AMPK in the regulation of cell proliferation and apoptosis by $\mathrm{p} 53$, and in the inhibition of cell growth and protein synthesis through inactivation of mTOR (Wang and Guan, 2009). In line with this hypothesis, clinical studies reported that low AMPK or pAMPK expression is related to a worse survival in several human malignancies (Hadad et al, 2009; Buckendahl et al, 2011; William et al, 2012). In CRC, however, Baba et al (2010) did not observe a significant impact of the activation of AMPK on survival. Notably, that study considered CRC patients at every stage and patients with stage IV CRC were only $14 \%$ of the study population. The study also showed that activation of the AMPK pathway had a significant impact on survival only in the subpopulation of patients that had a pMAPK3/1-positive tumour, representing $30 \%$ of the total samples analysed (Baba et al, 2010). Thus it is possible that variables including tumour stage and levels of pMAPK3/1 expression might explain the discrepancy between these two studies regarding the prognostic significance of $\mathrm{pAMPK}$.

With regard to the possibility that AMPK activation could represent a predictive biomarker, it should be noted that the study design does not allow to assess this, as all patients received FOLFIRI plus bevacizumab. Interestingly, pACC status showed a significant association with PFS. This may be due to the strong association between pACC status and surgery or loco-regional treatment of metastasis, which may induce a difference in PFS between pACC-positive and -negative groups. The association between pathway status and radical treatment of metastasis may possibly be explained by the prognostic role of the AMPK pathway.

The low OS of pAMPK-negative patients in the absence of differences in terms of PFS after FOLFIRI-bevacizumab may suggest an impact of AMPK status on tumour response to subsequent lines of therapy. This observation is intriguing because of some preclinical studies that suggested that cancer cells may acquire a more aggressive phenotype during anti-angiogenic treatment (Ebos et al, 2009; Paez-Ribes et al, 2009). In clinical practice, when disease progression is observed it might be more rapid after such treatment, leading to a shortened post-progression survival time. This issue, however, remains highly controversial. In fact, a recent meta-analysis found no differences in the time from discontinuation of bevacizumab or placebo to disease progression and death (Miles et al, 2011), but conclusions have been criticised, due to the exclusion of several large studies with quite obvious discrepancies between PFS and OS from this meta-analysis (Potemski, 2011). Our findings-albeit limited by the small size of the study and its retrospective nature-suggest that high tissue AMPK expression is prognostic for improved OS in this cohort of mCRC patients.

\section{ACKNOWLEDGEMENTS}

This work was supported by a grant from AIRC (IG 2013 grant number 14295). We thank Prof. Alberto Amadori (University of Padova) for critical reading of the manuscript.

\section{REFERENCES}

Baba Y, Nosho K, Shima K, Meyerhardt JA, Chan AT, Engelman JA, Cantley LC, Loda M, Giovannucci E, Fuchs CS, Ogino S (2010) Prognostic significance of AMP-activated protein kinase expression and modifying effect of MAPK3/1 in colorectal cancer. Br J Cancer 103: 1025-1033.

Bohndiek SE, Kettunen MI, Hu DE, Brindle KM (2012) Hyperpolarized [13]C spectroscopy detects early changes in tumor vasculature and metabolism after VEGF neutralization. Cancer Res 72: 854-864.

Buckendahl AC, Budczies J, Fiehn O, Darb-Esfahani S, Kind T, Noske A, Weichert W, Sehouli J, Braicu E, Dietel M, Denkert C (2011) Prognostic impact of AMP-activated protein kinase expression in ovarian carcinoma: correlation of protein expression and GC/TOF-MS-based metabolomics. Oncol Rep 25: 1005-1012.

Cancer Genome Atlas Network (2012) Comprehensive molecular characterization of human colon and rectal cancer. Nature 487: 330-337. Collett D (1994) Modelling Survival Data in Medical Research. Chapman and Hall: London. 
Ebos JM, Lee CR, Kerbel RS (2009) Tumor and host-mediated pathways of resistance and disease progression in response to antiangiogenic therapy. Clin Cancer Res 15: 5020-5025.

Eisenhauer EA, Therasse P, Bogaerts J, Schwartz LH, Sargent D, Ford R, Dancey J, Arbuck S, Gwyther S, Mooney M, Rubinstein L, Shankar L, Dodd L, Kaplan R, Lacombe D, Verweij J (2009) New response evaluation criteria in solid tumours: revised RECIST guideline (version 1.1). Eur J Cancer 45: 228-247.

Faivre S, Demetri G, Sargent W, Raymond E (2007) Molecular basis for sunitinib efficacy and future clinical development. Nat Rev Drug Discov 6: 734-745.

Ferrara N (2010) Role of myeloid cells in vascular endothelial growth factor-independent tumor angiogenesis. Curr Opin Hematol 17: 219-224.

Hadad SM, Baker L, Quinlan PR, Robertson KE, Bray SE, Thomson G, Kellock D, Jordan LB, Purdie CA, Hardie DG, Fleming S, Thompson AM (2009) Histological evaluation of AMPK signalling in primary breast cancer. BMC cancer 9: 307.

Hardie DG, Ross FA, Hawley SA (2012) AMPK: a nutrient and energy sensor that maintains energy homeostasis. Nat Rev Mol Cell Biol 13: 251-262.

Heath VL, Bicknell R (2009) Anticancer strategies involving the vasculature. Nat Rev Clin Oncol 6: 395-404.

Hurwitz H, Fehrenbacher L, Novotny W, Cartwright T, Hainsworth J, Heim W, Berlin J, Baron A, Griffing S, Holmgren E, Ferrara N, Fyfe G, Rogers B, Ross R, Kabbinavar F (2004) Bevacizumab plus irinotecan, fluorouracil, and leucovorin for metastatic colorectal cancer. New Engl J Med 350: 2335-2342.

Jessup JM, McGinnis LS, Steele Jr GD, Menck HR, Winchester DP (1996) The National Cancer Data Base. Report on colon cancer. Cancer 78: 918-926.

Jubb AM, Harris AL (2010) Biomarkers to predict the clinical efficacy of bevacizumab in cancer. Lancet Oncol 11: 1172-1183.

Kabbinavar FF, Hambleton J, Mass RD, Hurwitz HI, Bergsland E, Sarkar S (2005) Combined analysis of efficacy: the addition of bevacizumab to fluorouracil/leucovorin improves survival for patients with metastatic colorectal cancer. J Clin Oncol 23: 3706-3712.

Kang BW, Jeong JY, Chae YS, Lee SJ, Lee YJ, Choi JY, Lee IK, Jeon SW, Bae HI, Lee da K, Kwon OK, Chung HY, Yu W, Kim JG (2012) Phosphorylated AMP-activated protein kinase expression associated with prognosis for patients with gastric cancer treated with cisplatin-based adjuvant chemotherapy. Cancer Chemother Pharmacol 70: 735-741.

Keunen O, Johansson M, Oudin A, Sanzey M, Rahim SA, Fack F, Thorsen F, Taxt T, Bartos M, Jirik R, Miletic H, Wang J, Stieber D, Stuhr L, Moen I, Rygh CB, Bjerkvig R, Niclou SP (2011) Anti-VEGF treatment reduces blood supply and increases tumor cell invasion in glioblastoma. Proc Natl Acad Sci USA 108: 3749-3754.

Lambrechts D, Lenz HJ, de Haas S, Carmeliet P, Scherer SJ (2013) Markers of response for the antiangiogenic agent bevacizumab. J Clin Oncol 31: 1219-1230.

Lin D, Wei LJ, Ying Z (1993) Checking the cox model with cumulative sums of martingale-based residuals. Biometrika 80: 557-572.

Mehta S, Hughes NP, Buffa FM, Li SP, Adams RF, Adwani A, Taylor NJ, Levitt NC, Padhani AR, Makris A, Harris AL (2011) Assessing early therapeutic response to bevacizumab in primary breast cancer using magnetic resonance imaging and gene expression profiles. J Natl Cancer Inst Monogr 2011: 71-74.

Miles D, Harbeck N, Escudier B, Hurwitz H, Saltz L, Van Cutsem E, Cassidy J, Mueller B, Sirzen F (2011) Disease course patterns after discontinuation of bevacizumab: pooled analysis of randomized phase III trials. J Clin Oncol 29: $83-88$.

Nardo G, Favaro E, Curtarello M, Moserle L, Zulato E, Persano L, Rossi E, Esposito G, Crescenzi M, Casanovas O, Sattler UG, Mueller-Klieser W, Biesalski B, Thews O, Canese R, Iorio E, Zanovello P, Amadori A, Indraccolo S (2011) Glycolytic phenotype and AMP kinase modify the pathologic response of tumor xenografts to VEGF neutralization. Cancer Res 71: 4214-4225.

Paez-Ribes M, Allen E, Hudock J, Takeda T, Okuyama H, Vinals F, Inoue M, Bergers G, Hanahan D, Casanovas O (2009) Antiangiogenic therapy elicits malignant progression of tumors to increased local invasion and distant metastasis. Cancer Cell 15: 220-231.

Potemski P (2011) Is the postprogression survival time really not shortened in the bevacizumab-containing arms of phase III clinical trials? J Clin Oncol 29: e384-e385.

Price TJ, Hardingham JE, Lee CK, Weickhardt A, Townsend AR, Wrin JW, Chua A, Shivasami A, Cummins MM, Murone C, Tebbutt NC (2011) Impact of KRAS and BRAF gene mutation status on outcomes from the phase III AGITG MAX trial of capecitabine alone or in combination with bevacizumab and mitomycin in advanced colorectal cancer. J Clin Oncol 29: $2675-2682$.

Saltz LB, Clarke S, Diaz-Rubio E, Scheithauer W, Figer A, Wong R, Koski S, Lichinitser M, Yang TS, Rivera F, Couture F, Sirzen F, Cassidy J (2008) Bevacizumab in combination with oxaliplatin-based chemotherapy as first-line therapy in metastatic colorectal cancer: a randomized phase III study. J Clin Oncol 26: 2013-2019.

Sanchez-Cespedes M, Parrella P, Esteller M, Nomoto S, Trink B, Engles JM, Westra WH, Herman JG, Sidransky D (2002) Inactivation of LKB1/STK11 is a common event in adenocarcinomas of the lung. Cancer Res 62: 3659-3662.

Shackelford DB, Shaw RJ (2009) The LKB1-AMPK pathway: metabolism and growth control in tumour suppression. Nat Rev Cancer 9: 563-575.

Tsavachidou-Fenner D, Tannir N, Tamboli P, Liu W, Petillo D, Teh B, Mills GB, Jonasch E (2010) Gene and protein expression markers of response to combined antiangiogenic and epidermal growth factor targeted therapy in renal cell carcinoma. Ann Oncol 21: 1599-1606.

Wang W, Guan KL (2009) AMP-activated protein kinase and cancer. Acta Physiol 196: 55-63.

William WN, Kim JS, Liu DD, Solis L, Behrens C, Lee JJ, Lippman SM, Kim ES, Hong WK, Wistuba II, Lee HY (2012) The impact of phosphorylated AMP-activated protein kinase expression on lung cancer survival. Ann Oncol 23: 78-85.

Zheng L, Yang W, Wu F, Wang C, Yu L, Tang L, Qiu B, Li Y, Guo L, Wu M, Feng G, Zou D, Wang H (2013) Prognostic significance of AMPK activation and therapeutic effects of metformin in hepatocellular carcinoma. Clin Cancer Res 19: 5372-5380.

This work is published under the standard license to publish agreement. After 12 months the work will become freely available and the license terms will switch to a Creative Commons AttributionNonCommercial-Share Alike 3.0 Unported License.

Supplementary Information accompanies this paper on British Journal of Cancer website (http://www.nature.com/bjc) 